American Journal of Biochemistry and Biotechnology 10 (1): 31-39, 2014

ISSN: 1553-3468

(C) 2014 E. Takai et al., This open access article is distributed under a Creative Commons Attribution

(CC-BY) 3.0 license

doi:10.3844/ajbbsp.2014.31.39 Published Online 10 (1) 2014 (http://www.thescipub.com/ajbb.toc)

\title{
SCANNING ELECTRON MICROSCOPE IMAGING OF AMYLOID FIBRILS
}

\author{
${ }^{1}$ Eisuke Takai, ${ }^{1}$ Gai Ohashi, ${ }^{2}$ Ryuichi Ueki, \\ ${ }^{1}$ Yoichi Yamada, ${ }^{2}$ Jun-Ichi Fujita and ${ }^{1}$ Kentaro Shiraki \\ ${ }^{1}$ Faculty of Pure and Applied Science, University of Tsukuba, 1-1-1 Tennodai, Tsukuba, Ibaraki 305-8573, Japan \\ ${ }^{2}$ Tsukuba Research Center for Interdisciplinary Materials Science (TIMS), \\ Graduate School of Pure and Applied Sciences, University of Tsukuba, 1-1-1 Tennodai, Tsukuba 305-8573, Japan
}

Received 2014-02-06; Revised 2014-02-20; Accepted 2014-03-1

\begin{abstract}
This study demonstrated the applicability of Scanning Electron Microscopy (SEM) for the observation of amyloid fibrils without staining. As model specimens, two types of amyloid fibrils with different shapes and chemical compositions were controllably synthesized from hen lysozyme. The apparent fibril widths in the SEM images were considerably larger than the original diameters analyzed by the conventional techniques of Transmission Electron Microscopy (TEM) and Atomic Force Microscopy (AFM). Although this broadening, which depends on the chemical nature of the fibril, is not desirable for detailed imaging, it makes SEM sensitive to fibrils several micrometers in length and as thin as 3.5 $\mathrm{nm}$. Note that the sensitivity also contributed to clearly distinguishing amyloid fibrils from salt microcrystals in SEM images. These results suggest the considerable applicability of SEM for the imaging of amyloid fibrils, even in contaminated samples.
\end{abstract}

Keywords: Amyloid Fibrils, Scanning Electron Microscopy, Lysozyme

\section{INTRODUCTION}

Amyloid fibrils are linear beta-sheet-rich aggregates of protein that form spontaneously both in vivo and in vitro. Amyloid fibrils are of considerable interest because amyloidogenesis has often been linked to fatal degenerative disorders, such as Alzheimer's and Parkinson's diseases. However, the well-defined structure and biocompatibility of amyloid fibrils have also suggested new functional materials (Knowles and Buehler, 2011), such as amyloid fibrils attached to yellow fluorescent protein (Hamada et al., 2008), insulin amyloids as a biomaterial for cell culture surfaces (Sakono et al., 2011) and templates of peptide fibres for metal nanowire (Reches and Gazit, 2003).

Various measurement methods for amyloid fibrils have been developed, including (i) spectroscopic analysis by specific dyes, such as Congo red and thioflavin T, which are selectively adsorbed on the amyloid fibrils, (ii) spectroscopic determination of the beta-sheet structure in amyloid fibrils, such as circular dichroism and infrared spectra, (iii) binding and elongation analyses of fibrils by calorimetry (Kardos et al., 2004; Sasahara et al., 2007; Morel et al., 2010) and (iv) light, neutron and x-ray scattering to detect the solution structure of amyloid fibrils. These methods provide an average ensemble picture of amyloid fibrils (Nilsson, 2004). By contrast, Atomic Force Microscopy (AFM) and Transmission Electron Microscopy (TEM) can probe amyloid fibrils at the molecular level (Chamberlain et al., 2000; Ikeda and Morris, 2002; Khurana et al., 2003; Relini et al., 2004; Arimon et al., 2005; Lashuel and Wall, 2005; Anderson et al., 2006; Adamcik et al., 2010; Bayliss et al., 2009; Karakas et al., 2010). To our knowledge, Scanning Electron Microscopy (SEM) has rarely been used for imaging amyloid fibrils, although it possesses a Corresponding Author: Kentaro Shiraki, Faculty of Pure and Applied Science, University of Tsukuba, 1-1-1 Tennodai, Tsukuba, Ibaraki 305-8573, Japan Tel: +81-29-853-5306 Fax: +81-29-853-5215 
relatively high resolution of several nanometers and high usability through the detection of secondary electron emissions from specimen samples.

In this study, we demonstrated the imaging of amyloid fibrils by SEM. The results were compared with those of the well-used methods of TEM and AFM. Two types of amyloid fibrils were produced from Hen Egg White Lysozyme (HEWL) (Morshedi et al., 2007) with different shapes and chemical compositions. As expected, the apparent fibril width in the SEM image was broadened compared with the height in the AFM image. This broadening, however, provides a high detectability of amyloid fibrils with diameters as thin as $3.5 \mathrm{~nm}$. The chemical differences in the amyloid fibril are also reflected in the broadening of the image, which can thus be utilised for chemical-sensitive measurements.

\section{MATERIALS AND METHODS}

\subsection{Materials}

HEWL, sodium Chloride $(\mathrm{NaCl})$, potassium Chloride $(\mathrm{KCl})$ and sodium phosphate were from Nacalai Tesque Inc. (Tokyo, Japan). Thioflavin-T (ThT), sodium Hydroxide $(\mathrm{NaOH})$ and Hydrochloric Acid $(\mathrm{HCl})$ were from Wako Pure Chemical Industries, Ltd. (Osaka, Japan).

$\mathrm{S}$-amyloid was formed as follows. A stock solution containing $2.0 \mathrm{mg} \mathrm{mL}^{-1} \mathrm{HEWL}, 137 \mathrm{mM} \mathrm{NaCl}$ and 1.34 $\mathrm{mM} \mathrm{KCl}$ was prepared and adjusted to $\mathrm{pH} 2.0$ using $\mathrm{HCl}$. Then, the solution was incubated at $50^{\circ} \mathrm{C}$ for $4 \mathrm{~h}$ with continuous agitation by a stirrer.

A-amyloid was formed as follows. A stock solution containing $5.0 \mathrm{mg} \mathrm{mL}^{-1} \mathrm{HEWL}$ was prepared and adjusted to $\mathrm{pH} 1.0$ using $\mathrm{HCl}$. The solution was then incubated at $90^{\circ} \mathrm{C}$ for $9 \mathrm{~h}$ with continuous agitation by a stirrer.

The protein concentration was determined photometrically at $280 \mathrm{~nm}$ with an appropriate blank using a UV-vis spectrophotometer (ND-1000; NanoDrop Technologies Inc., Wilmington, DE). The extinction coefficient of $2.63 \mathrm{~mL} \mathrm{mg}^{-1} \mathrm{~cm}$ was used for HEWL.

\subsection{Thioflavin $T$ (ThT) Fluorescence Assay}

The growth of amyloid fibrils was monitored by fluorescence analysis with ThT. After fibril formation, $15 \mu$ Lof the sample solution was mixed with $1,485 \mu \mathrm{L}$ of $5 \mu \mathrm{M}$ ThT in $50 \mathrm{mM}$ Gly $\mathrm{NaOH}$ buffer ( $\mathrm{pH} 8.5$ ). The ThT fluorescence was monitored using a spectrofluorimeter (FP-6500; Jasco, Tokyo, Japan) with a 1-cm path-length quartz cell. The sample was excited at $440 \mathrm{~nm}(5 \mathrm{~nm}$ slit-width $)$ and the fluorescence intensity at $480 \mathrm{~nm}$ ( $5 \mathrm{~nm}$ slit-width) was monitored at $25^{\circ} \mathrm{C}$; the temperature was controlled using a temperature controller.

\subsection{Mass Spectra Measurement}

The mass spectra were obtained using matrix-assisted laser desorption/ionization time of flight mass spectrometry (MALDI-TOF MS) (UltrafleXtreme MALDI-TOF/TOF; Bruker Daltonics Inc., Billerica, USA). The amyloid was centrifuged from the sample solution and dissolved in pure water for the MALDITOF MS measurements. The matrix solution used for these measurements was a saturated $\alpha$-Cyano-4Hydroxycinnamic Acid (CHCA) solution in water/acetonitrile $1: 1 \mathrm{v} / \mathrm{v}$, diluted by a factor of 2 in the same solvent mixture. The sample was prepared by mixing $1.0 \mu \mathrm{L}$ of the protein solution with $1.0 \mu \mathrm{L}$ of the matrix solution directly on the sample probe and allowing the solution to dry at room temperature. Samyloid and A-amyloid were analyzed by MALDI-TOF$\mathrm{MS}$ in the positive-ion liner and reflect modes, respectively. Native HEWL was also analyzed by MALDI-TOF-MS in the positive-ion reflect mode.

\subsection{Imaging of Amyloid Fibrils by TEM, AFM and SEM}

Transmission Electron Microscopy (TEM) images of the amyloid fibrils were examined using stain (silicon tungstate) and a transmission microscope (JEM-1400; JEOL, Tokyo, Japan) with an acceleration voltage of 200 $\mathrm{keV}$ (Takai et al., 2014a). The sample solution was diluted 100-fold with pure water. Then, $2 \mu \mathrm{L}$ of the solution was negatively stained with $2 \mu \mathrm{L}$ of $2 \%$ (w/v) silicon tungstate solution. Next, $2 \mu \mathrm{L}$ of the stained solution was placed on a 150-mesh copper grid covered with a carbon-coated hydrophilic film. The solution on the grid was dried for a few minutes.

Atomic Force Microscopy (AFM) images of amyloid fibrils were examined using S-image (SII NanoTechnology Inc., Chiba, Japan) operating in tapping mode and a silicon cantilever (SI-DF20, SII Nanotechnology) with a tip curvature radius of $10 \mathrm{~nm}$ (Matsubara et al., 2009; Takai et al., 2014b). The sample solution was diluted 100 -fold with pure water. Subsequently, $1 \mu \mathrm{L}$ of the diluted samples was placed on freshly cleaved mica and dried in air for $10 \mathrm{~min}$. 
The Scanning Electron Microscopy (SEM) images of amyloid fibrils were examined using an SEM system (Hitachi S4800) with an acceleration voltage of $15 \mathrm{keV}$. The sample solution was diluted 100 -fold with pure water. Subsequently, $1 \mu \mathrm{L}$ of the diluted samples was placed on silicone and dried in air for $10 \mathrm{~min}$.

The fibril width values in the TEM and SEM images were measured by comparison with each scale bar. The fibril width values in the AFM image were measured from the transversal profiles of AFM topology. The fibril height values were also determined by the transversal profiles of AFM topology.

\section{RESULTS}

\subsection{Preparation of Amyloid Fibril From Self- Assembled Lysozyme (S-amyloid) and Acid- Hydrolysis Lysozyme (A-Amyloid)}

For the present study, we prepared two types of amyloid fibrils (S-amyloid and A-amyloid) from HEWL. S-amyloid is generated from HEWL incubated under acidic conditions ( $\mathrm{pH}$ 2) with saline under continuous agitation by a stirrer, as reported previously (Hirano et al., 2010; Takai et al., 2014c). Under this condition, amyloid fibrils are mainly formed from nonfragmented monomers of HEWL without hydrolysis. As expected, S-amyloid was characterised by a typical sigmoidal evolution of the Thioflavin $\mathrm{T}$ (ThT) fluorescent intensity with a lag time of 2-3 $\mathrm{h}$ (Fig. 1A). S-amyloid is a non-branched straight fibril with a height of approximately $5 \mathrm{~nm}$ and a length of approximately $500 \mathrm{~nm}$, with a helical structure (Fig. 1B and C). Figure 1D shows the histogram of the height of S-amyloid determined from an AFM image; the average diameter of the S-amyloid was $8 \pm 2 \mathrm{~nm}$, which is similar to the value previously reported for nonhydrolysed lysozyme fibril (Chamberlain et al., 2000). Notably, the histogram appears to exhibit two maxima centred at approximately 5 and $10 \mathrm{~nm}$, corresponding to the inner-fibril corrugation due to the helical structure of amyloid fibrils (Adamcik et al., 2010).
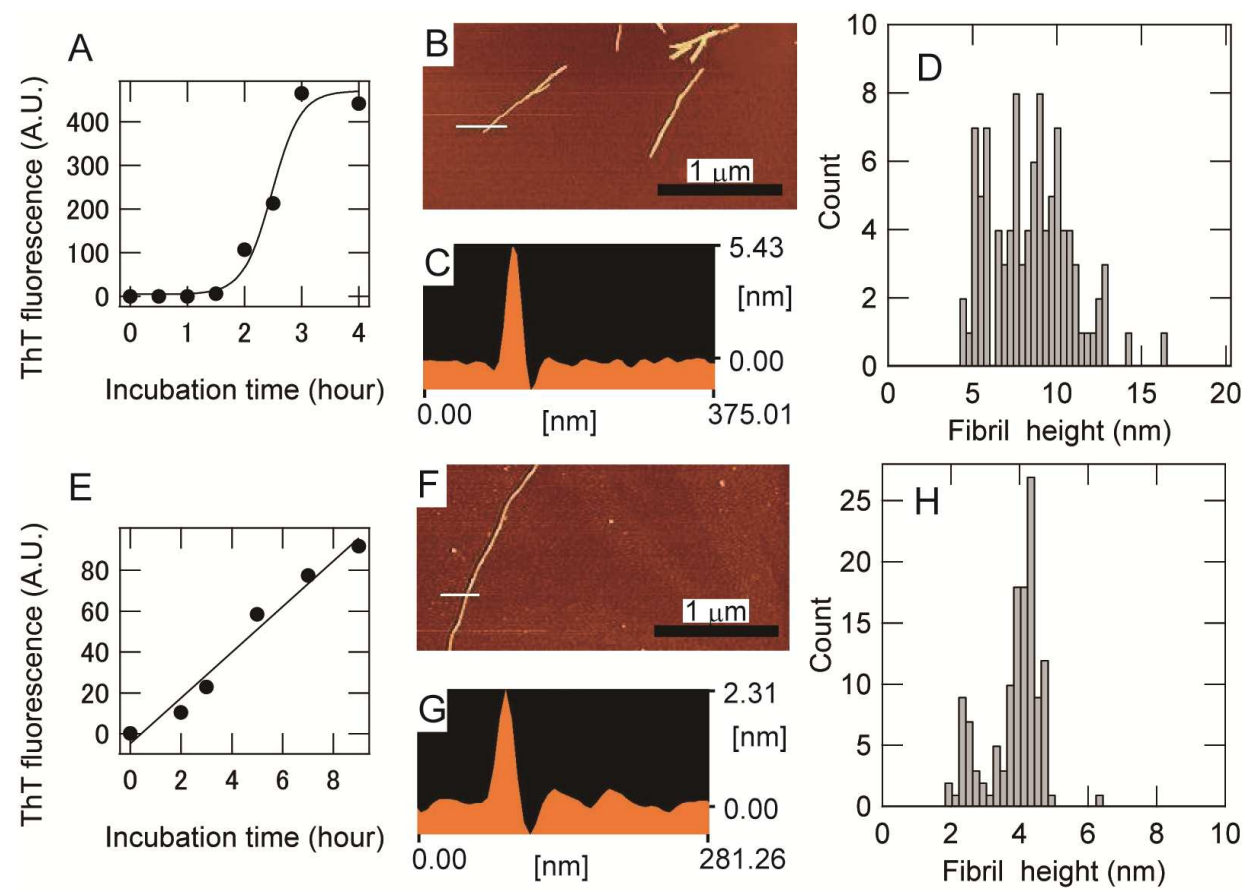

Fig. 1. Preparation and characterisation of S-amyloid and A-amyloid. (A) Growth of S-amyloid detected by thioflavin T (ThT) fluorescence. The continuous line though the data points was fitted with a sigmoidal curve. (B) AFM images of S-amyloid. The white line indicates the transversal profile of surface topology shown in C. (C) AFM topography image of S-amyloid, from which amyloid height was measured. (D) Histogram of S-amyloid AFM height (fibril diameter). (E-H) Figures for A-amyloid corresponding to the ones shown in Fig. (A-D). In E, the continuous line though the data points was fitted with a linear curve 
A-amyloid was similarly generated by incubation under more acidic conditions ( $\mathrm{pH} 1)$ at a higher temperature $\left(90^{\circ} \mathrm{C}\right)$, without saline but with continuous agitation by a stirrer. HEWL was hydrolysed under this condition (Lara et al., 2011). The peptide fragment of hydrolysed HEWL was immediately assembled into longer fibrils, characterised by the linear increase in ThT intensity with increasing incubation, without any lag time (Fig. 1E). In the AFM measurements, long and nonbranched fibrils of much greater than $1 \mu \mathrm{m}$ were observed with a height of approximately $2 \mathrm{~nm}$ (Fig. 1F and G). Figure $\mathbf{1 H}$ shows the histogram of the height of A-amyloid, with an average diameter of $4 \pm 1 \mathrm{~nm}$. The histogram also shows two maxima, at 2 and $4 \mathrm{~nm}$, corresponding to the helical shape of the fibril. The structure and dimensions agree with previously reported values (Lara et al., 2011).

Although they are generated from the same protein, S- and A-amyloid have different structural dimensions and chemical compositions. The MALDI-TOF MS spectra of S-amyloid showed two abundant peaks at $\mathrm{m} / \mathrm{z}$ 7153, which corresponds to the doubly protonated HEWL and at $\mathrm{m} / \mathrm{z} 14306$, which corresponds to the singly protonated HEWL (Fig. 2A). By contrast, the MALDI-TOF MS spectra of A-amyloid had many peaks below m/z 4000 (Fig. 2B). The MALDI-TOF MS spectra of native HEWL also had two abundant peaks at $\mathrm{m} / \mathrm{z}$ 7153 and 14306 (Fig. 2C). These results show that Samyloid was formed by nonfragmented monomers of HEWL without hydrolysis and A-amyloid was formed by aggregating peptide fragments of hydrolysed HEWL. Analysis using an amino acid analyzer (JLC500/V2,Japan Electron Optics Laboratory Ltd, Tokyo, Japan) revealed that A-amyloid contains fewer residues of Arg ( $\mathrm{pKa}$ 12.5) (Jin and Manabe, 2005), Lys (pKa 10.2) (Jin and Manabe, 2005) and His (pKa 6.0 (Edgcomb and Murphy, 2002) and more residues of Leu, Ile and Pro than native HEWL (data not shown), indicating that A-amyloid has less electrical capacity than S-amyloid. As described above, these two amyloid fibrils exhibited rather well-defined structures. Therefore, S-amyloid and A-amyloid are useful model fibrils for characterising the utility of SEM.

\subsection{Comparison with Fibril-Width Values in TEM, AFM and SEM Images}

Figure 3 displays large views of S-amyloid and Aamyloid imaged using TEM, AFM and SEM. In the TEM, AFM and SEM images, S-amyloid was consistently observed to form straight mature fibrils with lengths less than $1 \mu \mathrm{m}$ (Fig. 3A-C). However, considering the scale of the A-amyloid images, the fibrils shown by SEM were clearly different from those shown by TEM and AFM. Thus, we further analyzed the differences in the images of S-amyloid and A-amyloid by TEM, AFM and SEM.

Figure 4 A-C shows magnified images of a single Samyloid. The histogram of the apparent width of the fibrils in each image is presented in Fig. 4D-F. The TEM images of S-amyloid show clear edges of the fibrils and the centred value of the width was determined to be $13 \pm 7$ $\mathrm{nm}$ (Fig. 4A and D). The width determined from the TEM images, $13 \pm 7 \mathrm{~nm}$, was similar to the height of the fibril determined from the AFM images, $8 \pm 2 \mathrm{~nm}$. By contrast, the AFM images of S-amyloid had fine edges and exhibited wider widths of $38 \pm 8 \mathrm{~nm}$ (Fig. $4 \mathbf{B}$ and E). This phenomenon is a well-known broadening that reflects the finite curvature of the cantilever, approximately $10 \mathrm{~nm}$ (Matsubara et al., 2009).
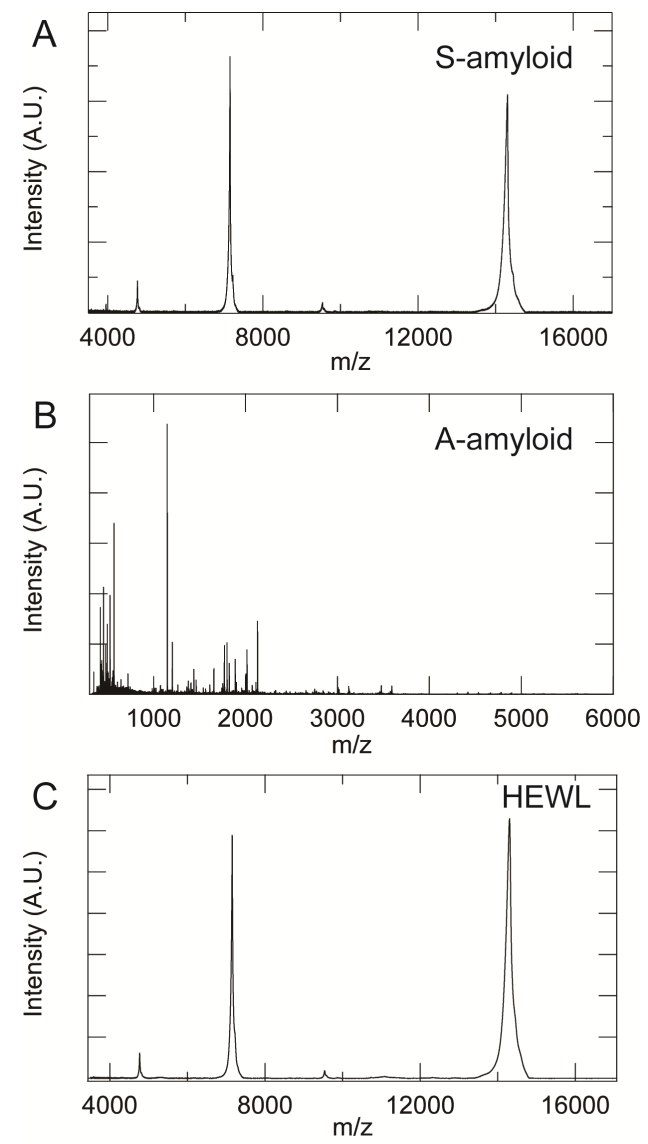

Fig. 2. MALDI-TOF MS spectra of S-amyloid (A), A-amyloid (B) and native HEWL as a control (C) 

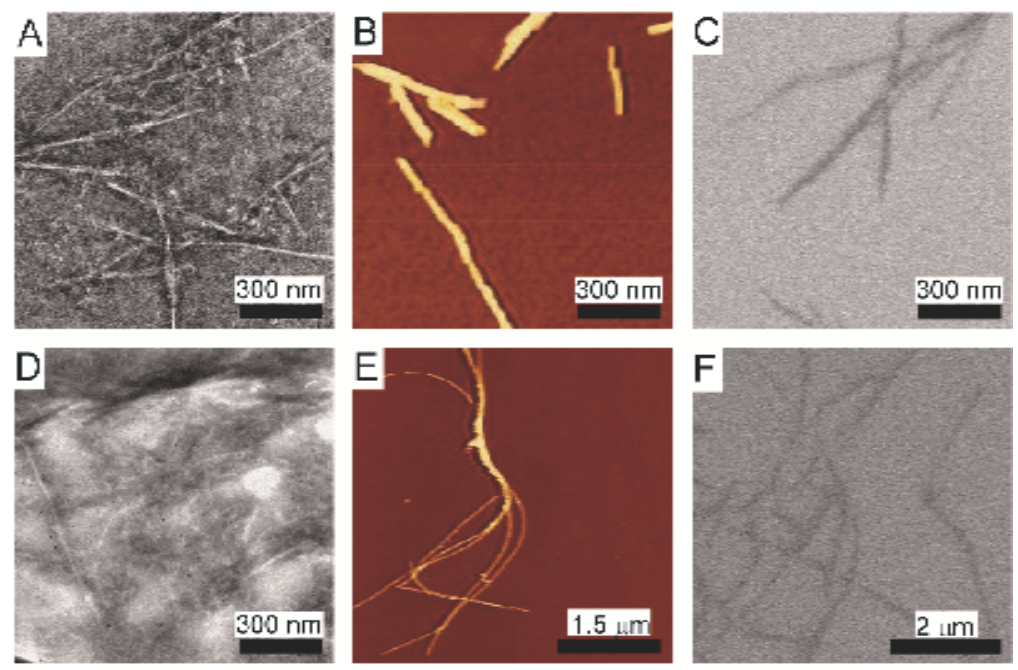

Fig. 3. Comparison of TEM, AFM and SEM. S-amyloid was monitored by TEM (A), AFM (B) and SEM (C). A-amyloid was monitored by TEM (D), AFM (E) and SEM (F)
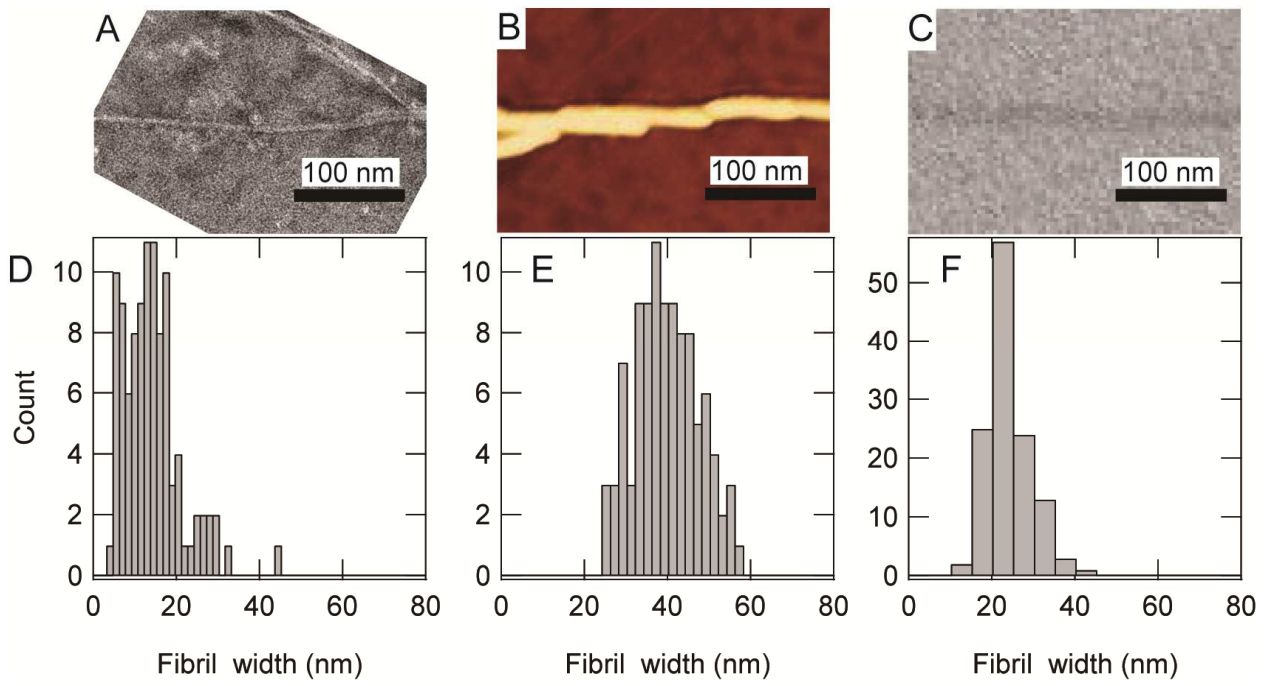

Fig. 4. Width of S-amyloid. microscopic images of S-amyloid by TEM (A), AFM (B) and SEM (C). Histogram of width of Samyloid by TEM (D), AFM (E) and SEM (F)

By contrast, the images of S-amyloid by SEM showed fuzzy edges due to its intrinsic lower resolution compared with TEM and AFM (Fig. 4C and F). The average width in the SEM images was as large as $22 \pm 5 \mathrm{~nm}$. During the SEM measurement, secondary electron emission from the amyloid fibril, which is an insulator, is less likely than that from the silicone plate, which is a semiconducting substrate, resulting in the dark appearance of the amyloid fibril in the
SEM image. Thus, there are multiple possible causes of the fuzzy appearance of the edge of the S-amyloid, including (i) the charging of S-amyloid induced by its lower secondary electron emission and (ii) the influence of counter ions $\left(\mathrm{Na}^{+}\right)$, which produce large secondary electron emissions.

A-amyloid was similarly characterised by TEM, AFM and SEM (Fig. 5). The results for A-amyloid were found to be similar to the results for S-amyloid; 
the TEM image provides an apparent width of $5 \pm 2$ $\mathrm{nm}$, which is close to the fibril height determined by AFM. The apparent width in the AFM image was again found to be as large as $33 \pm 4 \mathrm{~nm}$, reflecting the tip effect. A huge broadening of the apparent width, as large as $57 \pm 14 \mathrm{~nm}$, was observed in the SEM image of A-amyloid, although the original width of A-amyloid was much thinner than that of S-amyloid. This huge broadening of A-amyloid is probably due to the chemical nature of A-amyloid, which differs from the chemical nature of S-amyloid. A-amyloid should be more insulating than S-amyloid. A-amyloid consists of peptide fragments of hydrolysed HEWL and has fewer positively charged residues but more hydrophobic residues, whereas S-amyloid can contain plenty of non-beta-sheet side chains from nonfragmented HEWL, which can potentially be conducting. Thus, considering the different chemical natures of A-amyloid and S-amyloid, some possible causes of the huge broadening of A-amyloid in the SEM image include (i) more charging of A-amyloid and (ii) more influence from counter ions $\left(\mathrm{Na}^{+}\right)$.

\section{DISCUSSION}

The apparent width of amyloid fibrils by SEM was shown to be 7 to 10 times broader than the original value (Table 1). This broadening is not desirable for the detailed characterisation of the fibril structure, but the broadening of the apparent contrast can be utilised to increase the detectability of small fibrils. Indeed, in a large view, A-amyloid with diameters as small as $3.5 \mathrm{~nm}$ can be detected, as well as thicker fibrils (Fig. 6A). Although further characterisation of the relationship between the SEM appearance and the structure and composition of the amyloid fibrils is required, the high detectability of the small fibrils is useful for the screening of amyloid fibrils formed in vivo and in vitro.

Table 1. Diameter and width of amyloid fibrils measured by TEM, AFM and SEM

\begin{tabular}{llllll}
\hline & & S-amyloid & & A-amyloid \\
& & - & Average (nm) & \\
& & Replicates & Replicates & Average (nm) \\
\hline \multirow{2}{*}{ Height } & AFM & 99 & $8 \pm 2$ & 129 & $4 \pm 1$ \\
& TEM & 102 & $13 \pm 7$ & 96 & $5 \pm 2$ \\
& AFM & 100 & $38 \pm 8$ & 101 & $33 \pm 4$ \\
& SEM & 125 & $22 \pm 5$ & 123 & $57 \pm 14$ \\
\hline
\end{tabular}

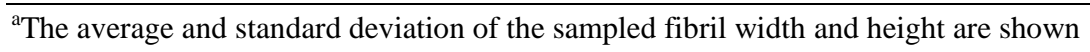
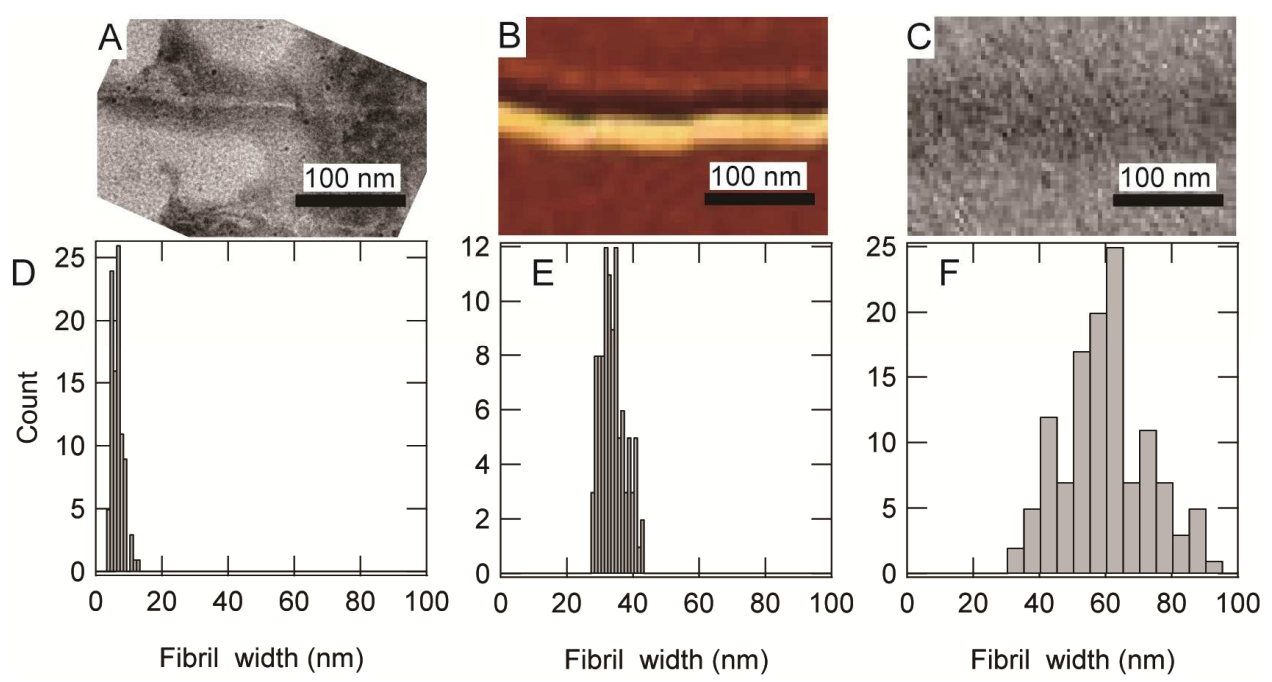

Fig. 5. Width of A-amyloid. microscopic images of A-amyloid by TEM (A), AFM (B) and SEM (C). Histogram of the width of Aamyloid by TEM (D), AFM (E) and SEM (F) 

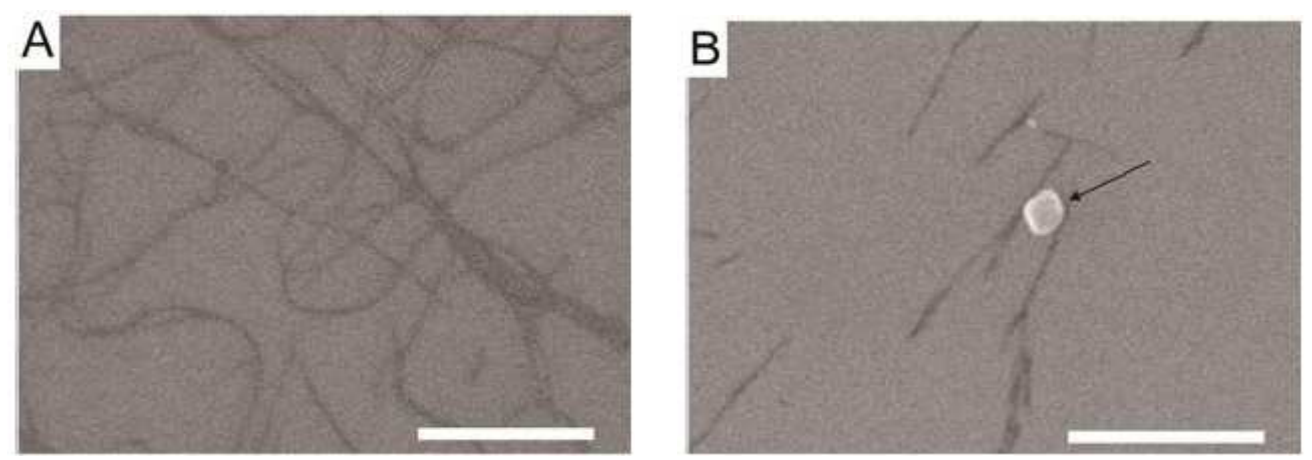

Fig. 6. (A) SEM image of A-amyloid. The scale bar is $3 \mu \mathrm{m}$. (B) SEM image of S-amyloid and a salt micro-crystal (black arrow). The scale bar is $1 \mu \mathrm{m}$

S-amyloid and small crystals of $\mathrm{NaCl}$ were clearly distinguished in the SEM image (Fig. 6B). Amyloid fibrils are usually dispersed in the solution with other solutes, such as inorganic salts and low-molecularweight compounds for $\mathrm{pH}$ buffering. These other solutes often disturb microscopic measurements in AFM and TEM. However, SEM clearly distinguishes amyloids from these contaminants because the secondary electron emission is sensitive to chemical natures (Fig. 6B). The easy distinction from contaminants suggests that SEM should be the first choice of microscopic measurement for amyloid fibril imaging.

\section{CONCLUSION}

This study examined SEM images of two types of amyloid fibrils compared with well-characterized AFM and TEM images. Although the resolution of SEM was found to be poor for insulating amyloid fibrils, even conventional SEM was able to detect amyloid fibrils as thin as $3.5 \mathrm{~nm}$ in width through broadening in the image. The broadening effect was also found to be dependent on the chemical composition of the fibril, which can be further utilized in chemical discrimination. Although further characterization of the dependence of the chemical properties on the apparent broadening will be required, the several advantages of SEM, in combination with other observations in solution, such as the TIRFM system (Ban et al., 2006; Ozawa et al., 2009; Yagi et al., 2010; Ozawa et al., 2011), cryo-TEM (Jimenez et al., 1999; 2001) and in-liquid AFM (Liu et al., 2012), contribute to the investigation of amyloid fibrils.

\section{ACKNOWLEDGEMENT}

The researchers acknowledge the Ministry of Education, Culture, Sports, Science and Technology
(MEXT), Japan, for supporting this study under Grant-in-Aid for Scientific Research (A) No. 23246063. The authors are grateful to the Chemical Analysis Division, Research Facility Centre for Science and Technology, University of Tsukuba, for amino acid analysis.

\section{REFERENCES}

Adamcik, J., J.M. Jung, J. Flakowski, P.D.L. Rios and G. Dietler et al., 2010. Understanding am-yloid aggregation by statistical analysis of atomic force microscopy images. Nature Nanotechnol., 5: 423428. DOI: $10.1038 /$ nnano.2010.59

Anderson, M., O.V. Bocharova, N. Makarava, L. Breydo and V.V. Salnikov et al., 2006. Polymorphism and ultrastructural organization of prion protein amyloid fibrils: An insight from high resolution atomic force microscopy. J. Mol. Biol., $\quad 358$ : 580-596. $\quad$ DOI: 10.1016/j.jmb.2006.02.007

Arimon, M., I. Diez-Perez, M.J. Kogan, N. Durany and E. Giralt et al., 2005. Fine structure study of Abeta142 fibrillogenesis with atomic force microscopy. FASEB J., 19: 1344-1346. DOI: 10.1096/fj.043137fje

Ban, T., K. Yamaguchi and Y. Goto, 2006. Direct observation of amyloid fibril growth, propagation and adaptation. Acc. Chem. Res., 39: 663-670. DOI: 10.1021/ar0500741

Bayliss, D.L., J.L. Walsh, G. Shama, F. Iza and M.G. Kong, 2009 Reduction and degradation of amyloid aggregates by a pulsed radio-frequency cold atmospheric plasma jet. New J. Phys., 11: 115024. DOI:10.1088/1367-2630/11/11/115024 
Chamberlain, A.K., C.E. MacPhee, J. Zurdo, L.A. Morozova-Roche and H.A. Hill et al., 2000. Ultrastructural organization of amyloid fibrils by atomic force microscopy. Biophys. J., 79: 32823293. DOI: 10.1016/S0006-3495(00)76560-X

Edgcomb, S.P. and K.P. Murphy, 2002. Variability in the $\mathrm{pKa}$ of histidine side-chains correlates with burial within proteins. Proteins-Structure Funct. Genet., 49: 1-6. DOI: 10.1002/prot.10177

Hamada, D., K. Tsumoto, M. Sawara, N. Tanaka and K. Nakahira et al., 2008. Effect of an amyloidogenic sequence attached to yellow fluorescent protein. Proteins-Structure Funct. Bioinformat., 72: 811-821. DOI: $10.1002 /$ prot.21971

Hirano, A., K. Uda, Y. Maeda, T. Akasaka and K. Shiraki, 2010. One-dimensional protein-based nanopar-ticles induce lipid bilayer disruption: Carbon nanotube conjugates and amyloid fibrils. Langmuir, 26: 17256-17259. DOI: $10.1021 / 1 \mathrm{a} 103615 \mathrm{~b}$

Ikeda, S. and V.J. Morris, 2002. Fine-stranded and particulate aggregates of heat-denatured whey proteins visualized by atomic force microscopy. Biomacromolecules, 3: 382-389. DOI: 10.1021/bm0156429

Jimenez, J.L., J.L. Guijarro, E. Orlova, J. Zurdo and C.M. Dobson et al., 1999. Cryo-electron microscopy structure of an SH3 amyloid fibril and model of the molecular packing. EMBO J., 18: 815-821. DOI: 10.1093/emboj/18.4.815

Jimenez, J.L., G. Tennent, M. Pepys and H.R. Saibil, 2001. Structural diversity of ex vivo amyloid fibrils studied by cryo-electron microscopy. J. Mol. Biol., 311: 241-247. DOI: 10.1006/jmbi.2001.4863

Jin, Y. and T. Manabe, 2005. High-efficiency protein extraction from polyacrylamide gels for molecular mass measurement by matrix-assisted laser desorption/ionization-time of flight-mass spectrometry. Electrophoresis, 26: 1019-1028. DOI: 10.1002/elps.200410187

Karakas, E., A. Munyanyi, L. Greene and M. Laroussi, 2010 Destruction of $\alpha$-synuclein based amyloid fibrils by a low temperature plasma jet. Appl. Phys. Lett., 97:143702. DOI:10.1063/1.3499277

Kardos, J., K. Yamamoto, K. Hasegawa, H. Naiki and Y. Goto, 2004. Direct measurement of the thermodynamic parameters of amyloid formation by isothermal titration calorimetry. J. Biol. Chem., 279: 55308-55314. DOI: 10.1074/jbc.M409677200
Khurana, R., C. Ionescu-Zanetti, M. Pope, J. Li and L. Nielson et al., 2003. A general model for amyloid fibril assembly based on morphological studies using atomic force microscopy. Biophys. J., 85: 1135-1144. DOI: 10.1016/S00063495(03)74550-0

Knowles, T.P.J. and M.J. Buehler, 2011. Nanomechanics of functional and pathological amyloid materials. Nature Nanotechnol., 6: 469-479. DOI: 10.1038/nnano.2011.102

Lara, C., J. Adamcik, S. Jordens and R. Mezzenga, 2011. General self-assembly mechanism converting hydrolyzed globular proteins into giant multistranded amyloid ribbons. Biomacromolecules, 12: 18681875. DOI: $10.1021 / \mathrm{bm} 200216 \mathrm{u}$

Lashuel, H.A. and J.S. Wall, 2005. Molecular electron microscopy approaches to elucidating the mechanisms of protein fibrillogenesis. Methods Mol Biol., 299: 81-101. DOI: 10.1385/1-59259-8749:081

Liu, P., S. Zhang, M.S. Chen, Q. Liu and C.X. Wang et al., 2012. Co-assembly of human islet Amyloid Poly-Peptide (hIAPP)/insulin. Chem. Commun., 48: 191-193. DOI: 10.1039/c1cc14285b

Matsubara, K., K.L. Kelly, N. Sakai and T. Tatsuma, 2009. Plasmon resonance-based photoelectrochemical tailoring of spectrum, morphology and orientation of Ag nanoparticles on TiO2 single crystals. J. Mater. Chem., 19: 55265532. DOI: 10.1039/B906795G

Morel, B., L. Varela and F. Conejero-Lara, 2010. The thermodynamic stability of amyloid fibrils studied by differential scanning calorimetry. J. Phys. Chem. B, 114: 4010-4019. DOI: 10.1021/jp9102993

Morshedi, D., N. Rezaei-Ghaleh, A. Ebrahim-Habibi, S. Ahmadian and M. Nemat-Gorgani, 2007. Inhibition of amyloid fibrillation of lysozyme by indole derivatives-possible mechanism of action. FEBS J., 274: 6415-6425. DOI: $10.1111 / \mathrm{j} .1742-$ 4658.2007.06158.x

Nilsson, M.R., 2004. Techniques to study amyloid fibril formation in vitro. Methods, 34: 151-160. DOI: 10.1016/j.ymeth.2004.03.012

Ozawa, D., Y. Kaji, H. Yagi, K. Sakurai and T. Kawakami et al., 2011. Destruction of amyloid fibrils of keratoepithelin peptides by laser irradiation coupled with amyloid-specific thioflavin T. J. Biol. Chem., 286: 10856-10863. DOI: 10.1074/jbc.M111.222901 
Ozawa, D., H. Yagi, T. Ban, A. Kameda and T. Kawakami et al., 2009. Destruction of amyloid fibrils of a beta2-microglobulin fragment by laser beam irradiation. J. Biol. Chem., 284: 1009-1017. DOI: $10.1074 /$ jbc.M805118200

Reches, M. and E. Gazit, 2003. Casting metal nanowires within discrete self-assembled peptide nanotubes. Science, 300: 625-627. DOI: 10.1126/science. 1082387

Relini, A., R. Rolandi, M. Bolognesi, M. Aboudan and G. Merlini et al., 2004. Ultra-structural organization of ex vivo amyloid fibrils formed by the apolipoprotein A-I Leu174Ser variant: An atomic force microscopy study. Biochim. Biophys. Acta, 1690: 33-41. DOI: 10.1016/j.bbadis.2004.04.007

Sakono, M., S. Akiyama, T. Zako, S. Sakaki and T. Waku et al., 2011. Application of two morphologically different fibrillar and filamentous insulin amyloids as a biomaterial for cell culture surfaces. React. Funct. Polym., 71: 324-328. DOI: 10.1016/j.reactfunctpolym.2010.10.012

Sasahara, K., H. Yagi, H. Naiki and Y. Goto, 2007. Heat-induced conversion of beta(2)-Microglobulin and hen egg-white lysozyme into amyloid fibrils. J. Mol. Biol., 372: 981-991. DOI: 10.1016/j.jmb.2007.06.088
Takai, E., G. Ohashi, T. Yoshida, K.M. Sorgjerd and T. Zako et al., 2014a. De-generation of amyloid- $\beta$ fibrils caused by exposure to low-temperature atmospheric-pressure plasma in aqueous solution, Appl. Phys. Lett., 104: 023701. DOI: 10.1063/1.4861842

Takai E., K. Uda, S. Matsushita, Y. Shikiya and Y. Yamada et al., 2014b. Cysteine inhibits amyloid fibrillation of lysozyme and directs the formation of small worm-like aggregates through non-covalent interactions, Biotechnol. Prog., DOI: 10.1002/btpr.1866

Takai E., K. Uda, T. Yoshida, T. Zako and M. Maeda et al., 2014c. Cysteine inhibits the fibrillisation and cytotoxicity of amyloid- $\beta 40$ and 42 : Implications for the contribution of the thiophilic interaction, Phys. Chem. Chem. Phys., 16: 3566-3572. DOI: 10.1039/C3CP54245A

Yagi, H., D. Ozawa, K. Sakurai, T. Kawakami and H. Kuyama et al., 2010. Laser-induced propagation and destruction of amyloid beta fibrils. J. Biol. Chem., 285: 19660-19667. DOI: 10.1074/jbc.M109.076505 